\title{
Regulation of Electrical Coupling Between Bio-pacemaker and Ventricular Myocytes on Autonomous Signal Propagation: A Simulation Study
}

\author{
Yacong $\mathrm{Li}^{1}$, Kuanquan Wang ${ }^{1}$, Qince Li ${ }^{1,3}$, Henggui Zhang ${ }^{2,3}$ \\ ${ }^{1}$ School of Computer Science and Technology, Harbin Institute of Technology, Harbin, China \\ ${ }^{2}$ School of Physics and Astronomy, the University of Manchester, Manchester, UK \\ 3 Peng Cheng Laboratory, Shenzhen, China
}

\begin{abstract}
Experiments showed that the expression of connexin Cx43 was suppressed in biological pacemakers (biopacemaker). Decreased Cx43 inhibited electrical coupling between bio-pacemakers and adjacent cardiac cells, which can maintain the synchronous pacemaking behaviour of bio-pacemaker. At the same time, moderate Cx43 is necessary to encourage autonomous electrical signal being propagated to non-rhythmic cardiac tissue. In this study, we simulated the electrical cell coupling among pacemaker myocytes (PMs) and ventricular myocytes in a two-dimensional idealized cardiac tissue model. We explored the effect of cell coupling pattern on the initiation of spontaneous signal in and the propagation capacity of the automaticity. If remaining cell coupling unchanged, the PMs tissue could not produce automaticity. When decreasing the coupling among PMs, PMs tissue presented synchronous pacemaking activity but the electrical signal could not propagate to the adjacent ventricular tissue. Then, according to the heterogeneity of intrinsic sinoatrial node, we divided PMs into central PMs and peripheral PMs. Only cell coupling of central PMs was decreased. In this way, PMs tissue could generate automatic pacemaking activity which could drive ventricular tissue. Our study might provide new sight into the electrical propagation mode of bio-pacemaker.
\end{abstract}

\section{Introduction}

The sinoatrial node (SAN) can produce automatic beatings and drive the whole heart because it can provide three factors: automaticity of single cardiac myocytes (CMs), electrical gap junction, and cardiac cellular networks (1). The traditional treatment of atrioventricular node dysfunction is installing an artificial electrical pacemaker in the ventricle. However, the patient has to face with complication risks during implanting surgery (2). And pediatric patient needs to replace the device with the change of the shape because of the fixed size of implantable pacemaker (3). Besides, the electrical pacemaker may be affected by electromagnetic interference in daily life (4). The biological pacemaker (bio-pacemaker) is hoped to substitute for electrical pacemaker and have the function of SAN when SAN failed to work. Our previous work has created a bio-pacemaker cell model based on a ventricular myocyte model (5). But if the automaticity of the single bio-pacemaker cell could produce synchronous pacing behaviour and propagate the spontaneous electrical signal to adjacent CMs still need to explore.

Biological experiments showed that the cell coupling between CMs decided the propagation between CMs. In SAN, cell coupling is little to maintain synchronization of both frequency and waveform (6). Bio-pacemaker experiments exhibited similar results. Bio-pacemaker can be transformed from ventricular myocytes (VMs) by gene therapy. Infecting transcription factor T-box 18 (TBX18) into VMs via adenoviral vectors made VMs show pacemaker cell morphology and spontaneous signal $(7,8)$. This kind of myocytes was called induced sinoatrial node (iSAN). Experiment results showed that iSAN expressed less $C \times 43$ gene than original VMs $(7,8)$. Cx43 is a kind of connexin, usually expressing in cardiac cells (9). The decrease of $C x 43$ weakened the electrical coupling between cells, which is helpful to maintain the pacemaking activity of the excitable cell. At the same time, intracellular $\mathrm{Ca}^{2+}$-oscillations in iSAN was asynchronous (10). That is to say, sparse $C x 43$ reduced coupling between cells and retarded the propagation of the electrical signal. Except for gene therapy, stem cells could also be induced to differentiate into pacemaker cells. For example, rat bone marrow stem cells (11) and adipose-derived stem cells (1214) can differentiate into pacemaker cells by infecting TBX18. Experiment results showed that Cx43 was also repressed in induced pacemaker (11), which was concordant with gene therapy experiments. A two-cell system showed that the spontaneous electrical signals produced by pacemaker cell could propagate to a quiescent 


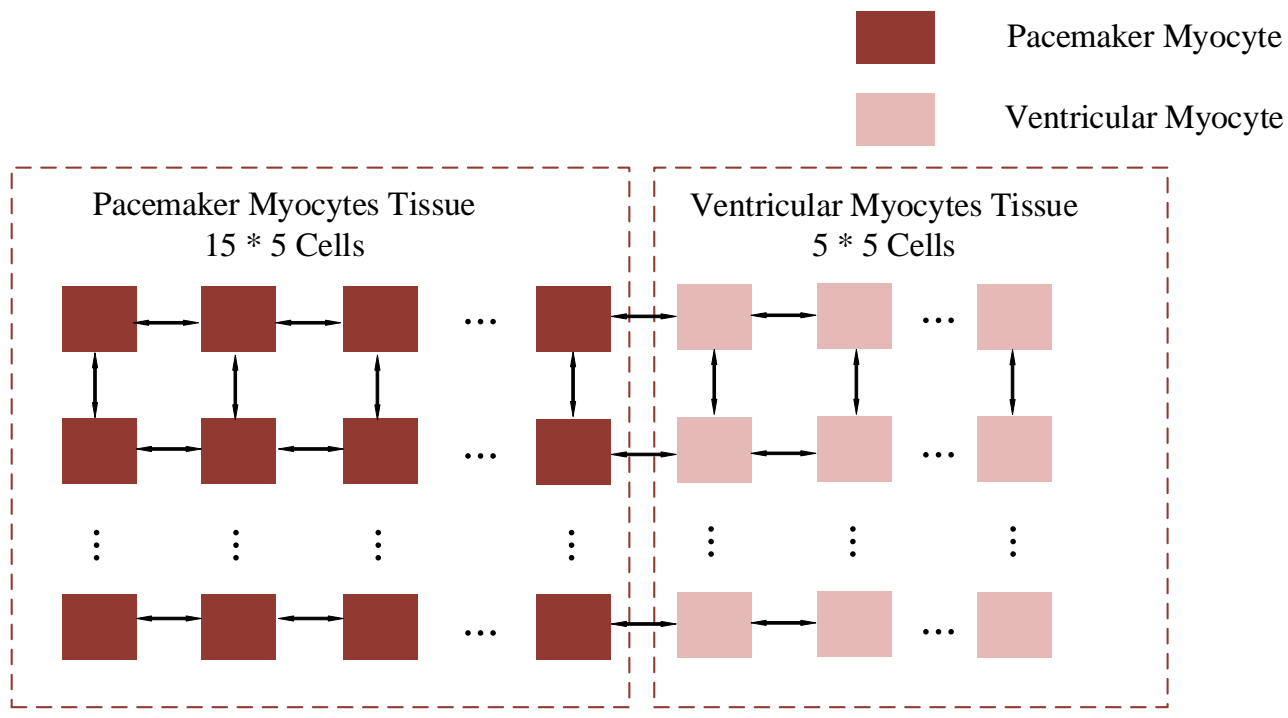

Figure. 1 The spatial distribution of a two-dimensional idealized pacemaker model. The size of ventricular myocytes tissue is $15 * 5$ cells connecting with $5 * 5$ pacemaker myocytes.

VM cell (15), which verified the ability of bio-pacemaker cells in propagation.

In this study, we constructed a two-dimensional (2D) idealized cardiac tissue model to simulate the effect of electrical coupling between bio-pacemaker cells and VMs on the synchronous pacemaking activity and propagation of the spontaneous electrical signal. We refactored the cell coupling by manipulating diffusion coefficient between CMs. Three different coupling patterns were engineered to illustrate how electrical coupling influenced pacemaking activity.

\section{Methods}

We designed an idealized electrophysiology cardiac tissue model where pacemaker myocytes (PMs) linked VMs. The model was used to simulate the propagation of spontaneous signal from PMs to VMs. The spatial construction of the idealized cardiac tissue was designed as Fig.1.

The electrophysiological behaviour of the CMs membrane could be described as follows

$$
\frac{\mathrm{dV}}{\mathrm{dt}}=-\frac{\mathrm{I}_{\text {ion }}}{\mathrm{C}_{\mathrm{m}}}+\nabla \cdot D \nabla V
$$

where $\mathrm{V}$ is membrane potential, $\mathrm{t}$ is time, $\mathrm{I}_{\mathrm{ion}}$ is the sum of all transmembrane ionic currents, and $\mathrm{C}_{\mathrm{m}}$ is cell capacitance, D is diffusion coefficient between cells. We simulated the different coupling pattern by manipulating D of CMs.

The $\mathrm{I}_{\text {ion }}$ of VMs is defined as follows (16):

$$
\mathrm{I}_{\text {ion }}=\mathrm{I}_{\mathrm{Na}}+\mathrm{I}_{\mathrm{K} 1}+\mathrm{I}_{\mathrm{to}}+\mathrm{I}_{\mathrm{Kr}}+\mathrm{I}_{\mathrm{Ks}}+\mathrm{I}_{\mathrm{CaL}}+\mathrm{I}_{\mathrm{NaCa}}
$$

$$
+\mathrm{I}_{\mathrm{NaK}}+\mathrm{I}_{\mathrm{pCa}}+\mathrm{I}_{\mathrm{pK}}+\mathrm{I}_{\mathrm{bCa}}+\mathrm{I}_{\mathrm{bNa}}
$$

According to our previous work (5), $\mathrm{I}_{\mathrm{ion}}$ of PMs could be described by

$$
\begin{aligned}
& \mathrm{I}_{\text {ion }}=\mathrm{I}_{\mathrm{Na}}+\mathrm{C}^{*} \mathrm{I}_{\mathrm{K} 1}+\mathrm{I}_{\mathrm{to}}+\mathrm{I}_{\mathrm{Kr}}+\mathrm{I}_{\mathrm{Ks}}+\mathrm{I}_{\mathrm{CaL}}+\mathrm{I}_{\mathrm{NaCa}} \\
& \quad+\mathrm{I}_{\mathrm{NaK}}+\mathrm{I}_{\mathrm{pCa}}+\mathrm{I}_{\mathrm{pK}}+\mathrm{I}_{\mathrm{bCa}}+\mathrm{I}_{\mathrm{bNa}}+\mathrm{I}_{\mathrm{f}}
\end{aligned}
$$

Where $\mathrm{c}$ is scale factor that simulates the suppression of $I_{K 1}$ current. The formulation of $I_{f}$ is listed in Ref. (17) and the formulations of other ionic currents could be referenced in (16).

\section{Results}

\subsection{Initiation of synchronous pacemaking activity}

When keeping D of whole tissue at the original value, the PMs could not produce any spontaneous impulse. On the one hand, the influence of non-rhythmic VMs inhibited the membrane potential of PMs from rising to activation potential of $I_{\text {Na. }}$. On the other hand, the interaction between PM cells restrained their membrane potential mutually. These two factors were responsible for the failure of synchronous pacemaking activity.

According to gene therapy experiments $(7,8)$, we decreased the cell coupling among PM cells to $1 \%$. Results showed that the single PM cell produced automatic pacemaking activity, and PM tissue expressed synchronous pacemaking activity. Due to the effect of nonautonomous VMs, the PMs depolarized gradually from 
A

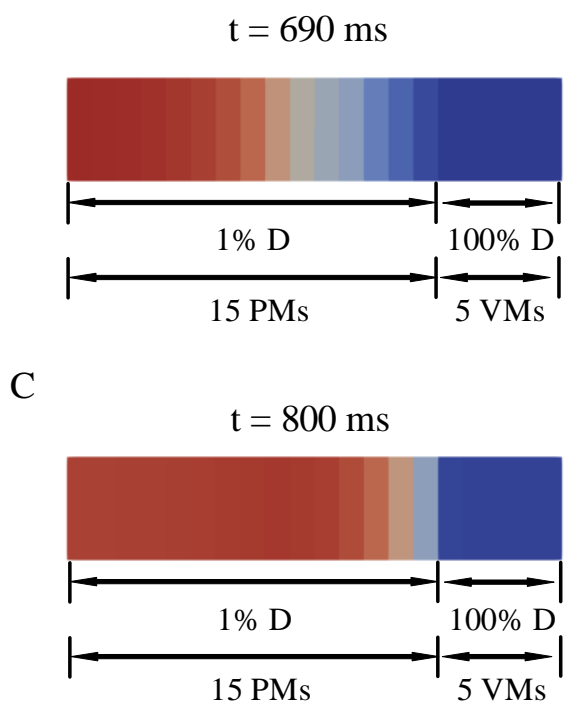

B

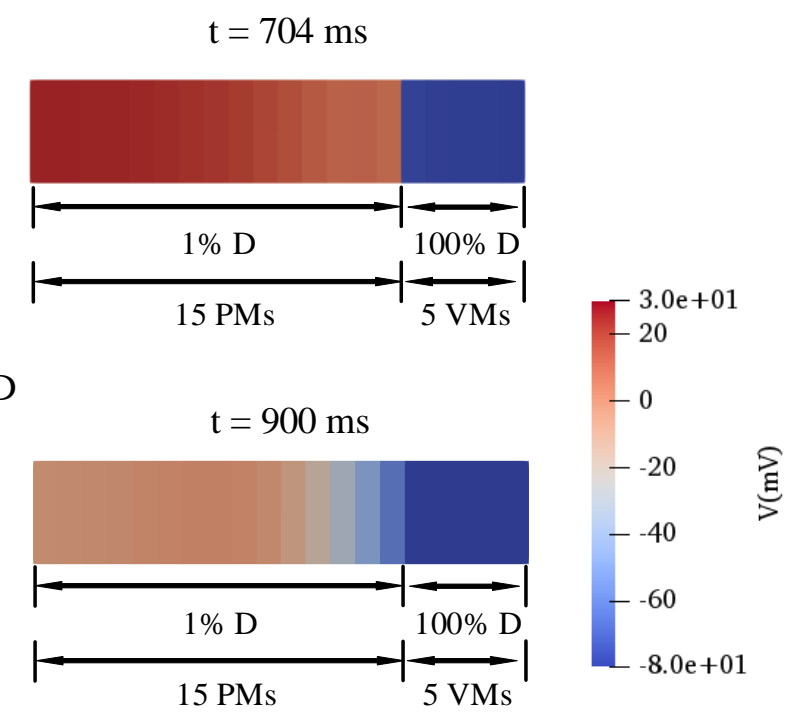

Figure. 2 The snapshots of conduction of spontaneous action potentials in idealized cardiac tissue when cell coupling of all PMs decreased to 1\%. A-D reflects the voltage of each single cardiac cell when the simulation time is $690,704,800,900 \mathrm{~ms}$ respectively.

left to right (Fig. 2A and 2B) and repolarized gradually from right to left (Fig. 2C and 2D). However, the spontaneous potential of PMs could not drive the VMs because of their weak coupling.

\subsection{Propagation of automaticity}

SAN has heterogeneity that may relate to different electrotonic coupling (18). The cell coupling of peripheral SAN cells is greater than the central SAN. We mimic the heterogeneity of SAN by modifying D according to the distance of PMs from VMs. The PMs was divided into central PMs (the first 10 PM cells) and peripheral PMs (the 5 PM cells close to VMs). As seen in Fig. 3, the D of central PMs were reduced to $1 \%$ where the D of peripheral PMs kept $100 \%$. On this condition, the spontaneous synchronous pacemaking activity was initiated at first (Fig. $3 \mathrm{~A}$ and $3 \mathrm{~B}$ ) and the automaticity propagated to VMs (Fig. $3 \mathrm{C}$ and $3 \mathrm{D})$.

\section{Conclusion}

In this study, we build a 2D cardiac tissue model and modified the cell coupling among CMs. The different coupling patterns were simulated and results showed that a low but enough level of electrical coupling among PM cells was required to provoke spontaneous pacemaking activity in PM tissue and propagate the automaticity to adjacent VMs. Our work verified that the pacemaker had a best pacemaking ability when being combined with cardiac tissue in the manner of how SAN combined with atrial tissue.

\section{Acknowledgements}

The work is supported by the National Natural Science Foundation of China (NSFC) under Grants No. 61572152, 61601143 and 81770328, the Science Technology and Innovation Commission of Shenzhen Municipality under Grants nos. JSGG20160229125049615 and JCYJ20151029173639477, China Postdoctoral Science Foundation under Grant nos.2015M581448.

\section{References}

1. Kleber AG, Rudy Y. Basic mechanisms of cardiac impulse propagation and associated arrhythmias. Physiol Rev. 2004;84(2):431-88.

2. Rosen MR, Robinson RB, Brink PR, Cohen IS. The road to biological pacing. Nature reviews Cardiology. 2011;8(11):656-66.

3. Cohen IS, Brink PR, Robinson RB, Rosen MR. The why, what, how and when of biological pacemakers. Nat Clin Pract Card. 2005;2(8):374-5.

4. Irnich $\mathrm{W}$, de Bakker JM, Bisping HJ. Electromagnetic interference in implantable pacemakers. Pacing Clin Electrophysiol. 1978;1(1):52-61. 
A

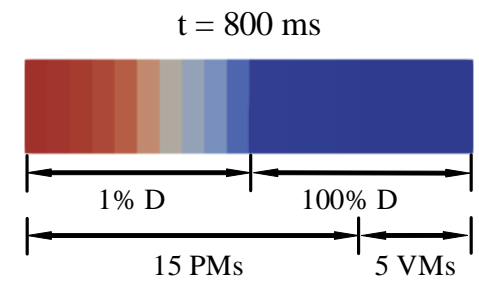

$\mathrm{C}$

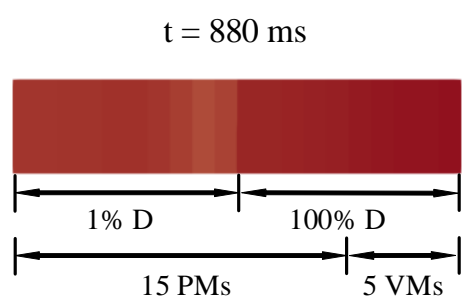

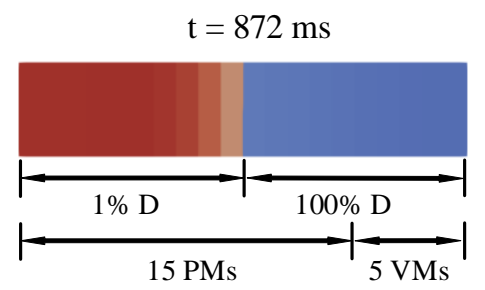

$\mathrm{D}$

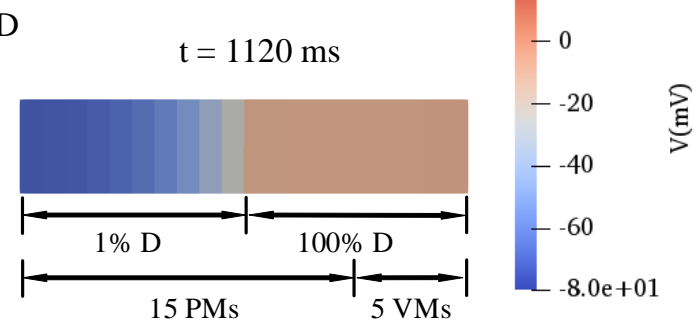

Figure. 3 The snapshots of conduction of spontaneous action potentials in idealized cardiac tissue when cell coupling of first 10 PMs decreased to $1 \%$. A-D reflects the voltage of each single cardiac cell when the simulation time is $800,872,880,1120 \mathrm{~ms}$ respectively.

5. Li Y, Wang K, Li Q, Luo C, Zhang H. Role of $\mathrm{I}_{\mathrm{f}}$ density on electrical action potential of bio-engineered cardiac pacemaker: a simulation study. Conference proceedings : Annual International Conference of the IEEE Engineering in Medicine and Biology Society IEEE Engineering in Medicine and Biology Society Annual Conference. 2019;2019:3995-8.

6. Wilders R, Verheijck EE, Kumar R, Goolsby WN, van Ginneken AC, Joyner RW, et al. Model clamp and its application to synchronization of rabbit sinoatrial node cells. Am J Physiol. 1996;271(5 Pt 2):H2168-82.

7. Kapoor N, Liang WB, Marban E, Cho HC. Direct conversion of quiescent cardiomyocytes to pacemaker cells by expression of Tbx18. Nat Biotechnol. 2013;31(1):54-+.

8. Hu YF, Dawkins JF, Cho HC, Marban E, Cingolani E. Biological pacemaker created by minimally invasive somatic reprogramming in pigs with complete heart block. Sci Transl Med. 2014;6(245).

9. Fromaget C, el Aoumari A, Gros D. Distribution pattern of connexin 43, a gap junctional protein, during the differentiation of mouse heart myocytes. Differentiation. 1992;51(1):9-20.

10. Kapoor N, Galang G, Marban E, Cho HC. Transcriptional suppression of connexin 43 by TBX18 undermines cell-cell electrical coupling in postnatal cardiomyocytes. J Biol Chem. 2011;286(16):14073-9.

11. Li Y, Yang M, Zhang G, Li L, Ye B, Huang C, et al. Transcription factor TBX18 promotes adult rat bone mesenchymal stem cell differentiation to biological pacemaker cells. Int J Mol Med. 2018;41(2):845-51.

12. Chen L, Deng ZJ, Zhou JS, Ji RJ, Zhang X, Zhang $\mathrm{CS}$, et al. Tbx18-dependent differentiation of brown adipose tissue-derived stem cells toward cardiac pacemaker cells. Mol Cell Biochem. 2017;433(1-2):61-77. 13. Yang M, Zhang GG, Wang T, Wang X, Tang YH, Huang $\mathrm{H}$, et al. TBX18 gene induces adipose-derived stem cells to differentiate into pacemaker-like cells in the myocardial microenvironment. Int $\mathbf{J}$ Mol Med. 2016;38(5):1403-10.

14. Zhang J, Huang C. A new combination of transcription factors increases the harvesting efficiency of pacemakerlike cells. Mol Med Rep. 2019;19(5):3584-92.

15. Valiunas V, Kanaporis G, Valiuniene L, Gordon C, Wang HZ, Li L, et al. Coupling an HCN2-expressing cell to a myocyte creates a two-cell pacing unit. J PhysiolLondon. 2009;587(21):5211-26.

16. ten Tusscher KH, Panfilov AV. Alternans and spiral breakup in a human ventricular tissue model. American journal of physiology Heart and circulatory physiology. 2006;291(3):H1088-100.

17. Fabbri A, Fantini M, Wilders R, Severi S. Computational analysis of the human sinus node action potential: model development and effects of mutations. The Journal of physiology. 2017;595(7):2365-96.

18. Oren RV, Clancy CE. Determinants of heterogeneity, excitation and conduction in the sinoatrial node: a model study. PLoS computational biology. 2010;6(12):e1001041.

Address for correspondence.

Yacong $\mathrm{Li}$

Room 901, Xinjishu Building, Harbin Institute of Technology

Harbin, China, 150001

li_yacong@163.com 